\title{
Targeting Host Syntaxin-5 Preferentially Blocks Leishmania Parasitophorous Vacuole Development in Infected Cells and Limits Experimental Leishmania Infections
}

\author{
Johnathan Canton and Peter E. Kima \\ From the Department of Microbiology and Cell Science, \\ University of Florida, Gainesville, Florida
}

Our previous observations established a role for syntaxin-5 in the development of Leishmania parasitophorous vacuoles (LPVs). In this study, we took advantage of the recent identification of Retro-2, a small organic molecule that can cause the redistribution of syntaxin-5; we show herein that Retro-2 blocks LPV development within 2 hours of adding it to cells infected with Leishmania amazonensis. In infected cells incubated for 48 hours with Retro-2, LPV development was significantly limited; furthermore, infected cells harbored four to five times fewer parasites than infected cells incubated in vehicle alone. In vivo studies revealed that Retro-2 curbed experimental $L$. amazonensis infections in a dose-dependent manner. Retro-2 did not have any appreciable effect on the host cell physiological characteristics; furthermore, it had no apparent toxicity in experimental animals. An unexpected, but welcome, finding was that Retro- 2 inhibited the replication of Leishmania parasites in axenic cultures. This study is significant because it identifies an endoplasmic reticulum/Golgi SNARE as a potential target for the control of Leishmania infections; moreover, it suggests that small organic molecules can be identified that can selectively disrupt the vesicle fusion machinery that promotes the development of pathogencontaining compartments without exerting toxic effects on the host. (Am J Pathol 2012, 181:1348-1355; http://dx. doi.org/10.1016/j.ajpath.2012.06.041)

Leishmania parasites are internalized into membranebound compartments called parasitophorous vacuoles (LPVs) that share many characteristics with phagosomes. They undergo a maturation scheme that includes sequential fusion with early endosomal compartments, from which they acquire early endocytic markers, such as EEA1 and Rab5 ${ }^{1,2}$; then, they fuse with late endosomal compartments and acquire molecules, such as vacuolar ATPase (vATPase), lysosome-associated membrane protein (LAMP-1), and lysosomal proteases (eg, cathepsins). ${ }^{3,4}$ Recently, however, there is evidence that, compared with phagosomes, LPVs have more sustained interactions with vesicles that originate from the early secretory pathway. ${ }^{5-7}$ From early times after formation, LPVs display, on their limiting membrane, resident endoplasmic reticulum (ER) molecules (eg, calnexin) and soluble $\mathrm{N}$-ethylmaleimide-sensitive factor attachment protein receptors [SNAREs; eg, sec22b, p31/D12 (D12), and syntaxin-18 (STX18) or STX5] that mediate the fusion of early secretory pathway vesicles. It is not known what molecular interactions promote the sustained interactions of LPVs with early secretory pathway vesicles. Furthermore, the advantages that acquisition of molecules from the ER or early secretory pathway vesicles confers to LPVs or the parasites therein are unknown.

There are several well-studied molecule families that mediate vesicle fusion in eukaryotic cells; these molecule families include Rab proteins, tether proteins, and SNAREs. ${ }^{8,9}$ In the case of SNAREs, a subset of members of this family expressed on donor vesicles ( $v$-SNAREs or R-SNAREs) form complexes with cognate partners on target vesicles (t-SNAREs or Q-SNAREs) that provide some of the energy to complete the fusion of the apposed membranes. ${ }^{10}$ Although SNARE molecules function in complexes, there is evidence from gene deletion experiments and knockdown studies that individual SNAREs might be differentially dispensable in the fusion of some

Supported by the University of Florida Foundation.

Accepted for publication June 20, 2012.

Supplemental material for this article can be found at http://ajp. amjpathol.org or at http://dx.doi.org/10.1016/j.ajpath.2012.06.041.

Address reprint requests to Peter E. Kima, Ph.D., Department of Microbiology and Cell Science, University of Florida, Building 981, Box 110700 , Gainesville, FL 32611. E-mail: pkima@ufl.edu. 
vesicles. ${ }^{11-13}$ Alternately, some processes in cells that depend on vesicle fusion events are less affected by the absence of a single SNARE molecule compared with other processes in the same cell that might exhibit greater effects from the loss of that SNARE. Such examples can be found in studies on the role of ER in the phagocytosis of large particles; in one study, the expression of dominant-negative forms of sec22b preferentially inhibited the uptake of large particles by macrophages. ${ }^{14}$ Similar observations were made in studies on the uptake of polystyrene latex beads and Zymosan particles that included knockdown of sec22b and STX18. ${ }^{15,16}$ An observation from those studies is that, although the targeting of those ER/Golgi SNAREs had a significant effect on the uptake of large latex beads and Zymosan, it had minimal impact on processes in the early secretory pathway in which these SNAREs normally function. Complementary observations were made in studies on infection of cells with Legionella, in which sec22b engaged in noncognate pairing with plasma membrane SNAREs to promote the uptake of virulent Legionella compared with the uptake of nonvirulent bacteria. Knockdown of sec22b levels selectively inhibited live Legionella uptake. ${ }^{17}$ In a somewhat related process, it was recently shown that, although knockdown of sec22b inhibited antigen cross presentation in dendritic cells, it did not change the growth rate, viability, and phenotype of the cells that depend on processes in which sec22b plays a physiological role. ${ }^{18}$ Taken together, these studies imply that it is possible to disrupt some processes in cells that depend on vesicle fusion by targeting the functions of a single SNARE molecule without adversely affecting the physiological characteristics of the cell.

To determine the role of individual SNARE molecules in the interactions of vesicles within the endomembrane system, several approaches have been implemented. They include the expression of dominant-negative variants that compete with the native molecule, knockdown of SNAREs of interest to levels that render them limiting, ${ }^{7,12}$ and performance of experiments in cells from animals in which SNARE genes have been deleted. ${ }^{13}$ Recently, molecules from small-molecule libraries have been screened to identify molecules that can block processes that depend on vesicle fusion. ${ }^{19,20}$ This latter approach was implemented to identify small molecules that can block the retrograde transport of ricin in mammalian cells. ${ }^{21}$ Retro-1 and Retro-2 were identified and partially characterized as molecules that target the SNARE proteins STX6 and STX5, respectively. These small molecules exerted their functions in cultured cells and in the in vivo setting. Those studies strongly suggested that administration of Retro-1 and Retro-2 was not toxic to either cultured cells or mice.

In light of the observations that indicated that STX5 is displayed on LPVs and that the interactions of LPVs with early secretory vesicles could be disrupted by the knockdown of STX5, ${ }^{7}$ we assessed the effect of Retro-2 on Leishmania amazonensis infections. Retro-2 blocked LPV distention, inhibited parasite replication in infected cells, and limited the course of experimental infection after the administration of a single dose of Retro-2. An unex- pected, but welcome, observation was that Retro-2 had a specific effect on Leishmania parasites. Not unlike other lower eukaryotes, Leishmania parasites have fewer SNARE molecules; this might result in reduced SNARE redundancy, which apparently renders them more susceptible to targeting.

\section{Materials and Methods}

\section{Parasites, Cell Lines, and Animals}

The L. amazonensis (MHOM/BR/77/LTB0016) promastigotes [obtained from ATCC (Manassas, VA) and Dr. Diane McMahon-Pratt, Yale University (New Haven, CT)] were cultured in Schneider's Drosophila Medium (Gibco, Grand Island, NY) supplemented with 20\% heat-inactivated fetal bovine serum (Atlanta Biologicals, Lawrenceville, GA) and $10 \mu \mathrm{g} / \mathrm{mL}$ gentamicin (Gibco) and grown at $23^{\circ} \mathrm{C}$. Parasite pathogenicity was maintained by regular passage through mice.

The RAW264.7 murine macrophage-like cell line (obtained from ATCC) was maintained as previously described. ${ }^{6}$ Primary mouse macrophages were obtained from the peritoneal exudate of BALB/c mice stimulated with thioglycolate 4 days before macrophage recovery.

BALB/c mice at the age of 6 to 8 weeks (The Jackson Laboratory, Bar Harbor, ME) were maintained in specific pathogen-free conditions at the Association for Assessment and Accreditation for Laboratory Animal Care-accredited University of Florida, Gainesville, under the supervision of the Institutional Animal Care and Use Committee, in strict accordance with approved protocols.

\section{Antibodies, Immunofluorescence Labeling, and Imaging}

The STX5 antibody was obtained from Santa Cruz Biotechnology, San Jose, CA. The GM130 antibody was obtained from BD Biosciences, San Jose. The 1D4B antibody was obtained from the Developmental Studies Hybridoma Bank, lowa City, IA. Hybridoma clones 5C6 (antiCR3) and 2.4G2 (anti-FcRII) were obtained from ATCC; hybridoma supernatants were prepared in the laboratory. Alexa Fluor secondary antibodies were obtained from Molecular Probes, Carlsbad, CA.

Immunofluorescence labeling was performed as previously described. ${ }^{6}$ Labeled coverslips were examined on a Zeiss Axiovert $200 \mathrm{M}$ microscope with a plain neofluar $100 \times / 1.3$ oil-immersion objective. Images were captured with an AxioCam MRm camera controlled by AxioVision software (Zeiss). Image series over a defined Z-focus range were acquired and processed with three-dimensional deconvolution software supplied by AxioVision. An extended focus function was used to merge optical sections to generate the images presented in the figures.

\section{PV Measurement and Parasite Counts}

Z-stack images of PVs were acquired as previously described. Z-stack heights were selected to cover the entire 
host cell nucleus and any PVs being measured to ensure that the widest point of the host cell nucleus and PV was captured. Images were deconvoluted and collapsed using the Extended Focus tool on AxioVision. The Outline Spline tool in the AxioVision software was used to measure both the circumference of the LPV and the host cell nucleus (in micrometers). The circumference of each LPV was normalized by dividing by the circumference of the host cell nucleus. This ratio was presented as Relative PV Size.

Parasite counting was performed on the immunofluorescence microscope previously described. Infected cells were processed for immunofluorescence analyses, and the DAPI stain was used to visualize parasite nuclei. Parasite nuclei were enumerated at respective time points.

\section{LPS/Interferon- $\gamma$ Activation}

RAW264.7 cells were plated at $1.8 \times 10^{6}$ cells $/ \mathrm{mL}$ in 60-mm cell culture dishes in Dulbecco's modified Eagle's medium (Sigma-Aldrich, St. Louis, MO) supplemented with $10 \%$ heat-inactivated fetal bovine serum and $100 \mathrm{U}$ of penicillin-streptomycin (Dulbecco's modified Eagle's medium complete) and incubated for 24 hours at $37^{\circ} \mathrm{C}$ and $5.5 \% \mathrm{CO}_{2}$. After the 24-hour incubation, the medium was aspirated and replaced with Dulbecco's modified Eagle's medium complete supplemented with interferon- $\gamma$, at $100 \mathrm{U} / \mathrm{mL}$, and lipopolysaccharide (LPS), at 10 $\mu \mathrm{g} / \mathrm{mL}$, and placed back in the incubator for another 24-hour incubation. The supernatant fluid was then collected, and an enzyme-linked immunosorbent assay (ELISA) for IL-6 (BD Biosciences, OptEIA) was performed. Because some cells were lost during transfection, the ELISA results were normalized using the average number of cells per field from 20 fields counted from the plates before the supernatant was collected.

\section{Treatment of Infected Cells with Retro-2 and the Parasite Intoxication Assay}

Retro-2, 2-\{[(5-methyl-2-thienyl)methylene]amino\}-N-phenylbenzamide (5374762), 5322770, and 5322213 were obtained from ChemBridge Corporation (San Diego, CA). Either RAW264.7 or elicited peritoneal exudate cells (PECs) on coverslips were infected at a parasite/macrophage ratio of $10: 1$ for 2 hours before the addition of Retro-2 or other small molecules. A range of concentrations of each molecule was tried. Infections were stopped at the times indicated in the figures; coverslips were processed for the evaluation of LPV dimensions and parasite burden, as previously described..$^{6,7}$ For evaluation of direct effects on parasites, $1 \times 10^{7}$ promastigote forms were seeded in each well of a 24-well plate containing 1 $\mathrm{mL}$ of Schneider's Drosophila medium, supplemented as previously described. Wells were treated with vehicle [dimethylsulfoxide (DMSO)] or $25,50,75$, or $100 \mu \mathrm{mol} / \mathrm{L}$ Retro-2. Parasites were left to grow at $23^{\circ} \mathrm{C}$. Parasite counts for each well were counted every 24 hours for 9 days using a hemocytometer. Each group was performed in triplicate ( $n=3$ wells).

\section{Infection and Retro-2 Treatment of Mice}

BALB/c mice were infected in their hind feet with $2 \times 10^{6}$ stationary stage cultured $L$. amazonensis promastigotes. The course of infection in at least 10 mice was monitored by measurement of foot size using a dial-gauge caliper. At the indicated times, mice were sacrificed to determine parasite burdens at the site of infection by limiting dilution analysis, as previously described. ${ }^{22}$ For drug-treated groups, Retro-2 dosage was dissolved in DMSO and made up to the respective dose (100 or $20 \mathrm{mg} / \mathrm{kg}$ ) in one times sterile PBS. Mice weighed approximately $18 \mathrm{~g}$. Retro-2 in a $150-\mu \mathrm{L}$ volume was administered i.p. at 24 hours or 3 weeks after infection. Each drug-treated group consisted of 8 to 12 mice. The course of infection was monitored as previously indicated.

\section{Co-IP Data}

Cells grown on petri dishes were infected at a parasite/ macrophage ratio of 10:1. Plates to be processed for co-immunoprecipitation (co-IP) were lysed in $50 \mathrm{mmol} / \mathrm{L}$ Tris-HCL (pH 7.4), $15 \mathrm{mmol} / \mathrm{L}$ EDTA, $100 \mathrm{mmol} / \mathrm{L} \mathrm{NaCl}, 1$ $\mathrm{mmol} / \mathrm{L} \mathrm{N}$-ethylmaleimide, and $1 \%$ Triton $\mathrm{X}-100$ with the Roche Complete Mini (Roche, Indianapolis, IN). Lysis was performed for 30 minutes at $4^{\circ} \mathrm{C}$ with gentle rocking. Lysate was collected and spun down at 10,000 $\times g$ for 10 minutes. The cleared lysate, $1 \mathrm{mg}$, was adjusted to 500 $\mu \mathrm{L}$ with co-IP buffer $[50 \mathrm{mmol} / \mathrm{L}$ Tris-HCL $(\mathrm{pH} 7.4), 15$ $\mathrm{mmol} / \mathrm{L}$ EDTA, $100 \mathrm{mmol} / \mathrm{L} \mathrm{NaCl}, 1 \mathrm{mmol} / \mathrm{L} \mathrm{N}$-ethylmaleimide, and $0.1 \%$ Triton $X-100$ ]. A $50 \%$ slurry of protein $G$ beads, $25 \mu \mathrm{L}$ (Protein G 4-fast flow; Amersham, Pittsburgh, PA), was added to the lysate and placed at $4^{\circ} \mathrm{C}$ for 25 minutes. Protein $\mathrm{G}$ beads were removed, and $2 \mu \mathrm{g}$ of the appropriate antibody was added to the cleared lysate. Tubes were placed at $4^{\circ} \mathrm{C}$ with gentle rocking for 2 hours. A $50 \%$ slurry of Protein G beads, $75 \mu \mathrm{L}$, was added, and the tubes were placed back at $4^{\circ} \mathrm{C}$ for an additional hour. Beads were spun down and washed five times with $1 \mathrm{~mL}$ of co-IP buffer. Sample buffer (Laemmli) was added to the bead pellet after the final wash. Beads were boiled in sample buffer for 5 minutes. Beads were spun down, and the supernatant was saved for SDSPAGE.

Enrichment of LPVs was performed as previously described. ${ }^{23}$

\section{Statistics}

Data analysis and the generation of graphs were performed using Microsoft Excel and Sigmaplot software version 10.0 (Systat Software, Inc. San Jose, CA). Each data point is presented as the mean, with the SE indicated with $y$-error bars. Boxplots for PV size were generated in Sigma-Plot, and boxes represent the range of PV sizes for the given condition; each outlier is shown as a single point. Significance (indicated by an asterisk) was 

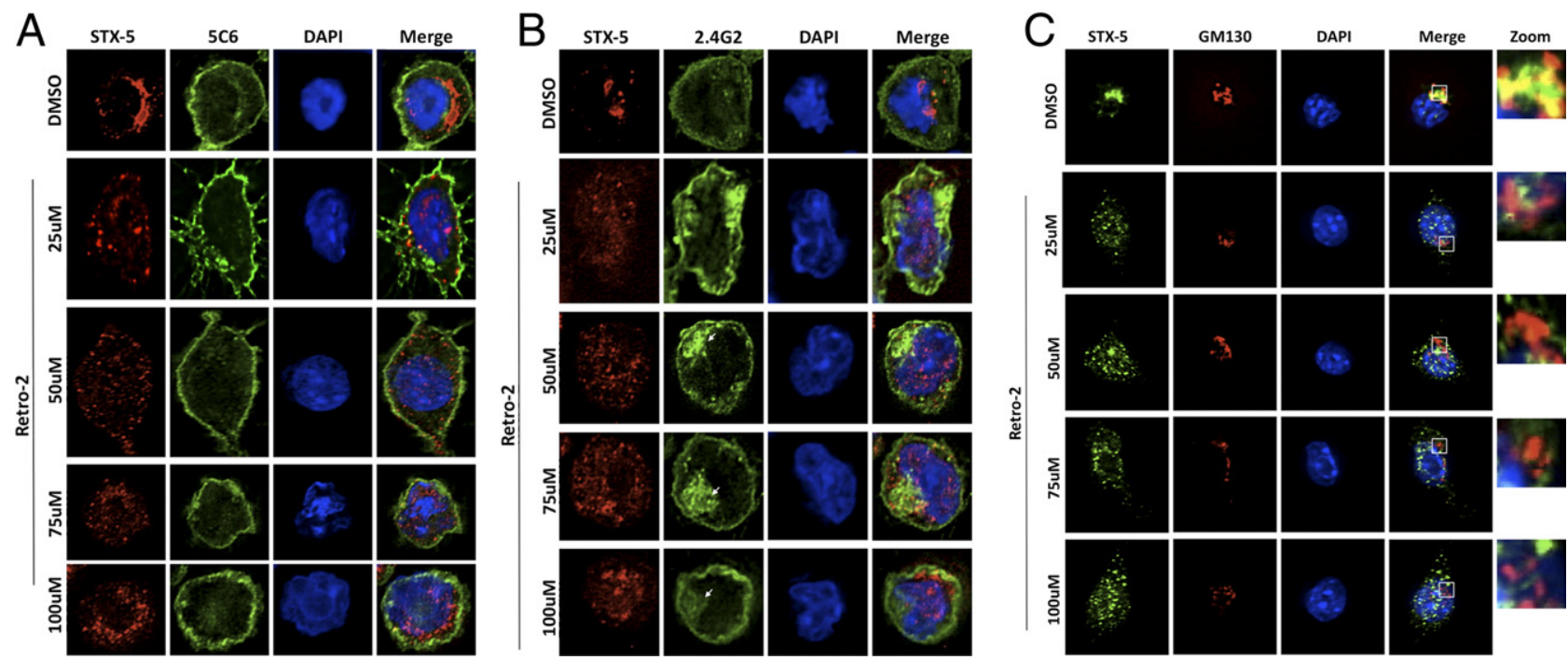

Figure 1. Retro-2 treatment results in mislocalization of STX5. RAW264.7 cells were cultured in increasing concentrations of Retro-2. By using antibodies to STX5 and CR3 (A), FCR (B), or GM130 (C), the distribution of these molecules was determined. The effect of Retro- 2 was compared with cells incubated with the vehicle (DMSO). Retention of FCR in a cellular compartment (B) is marked (arrows). Images are representative of at least three experiments.

assessed using the $t$-test, and two arrays were considered significantly different at $P \leq 0.05$.

\section{Results}

\section{Evaluation of the Effect of Retro-2 on Cells}

The initial studies on Retro-2 showed that it blocked ricin toxicity by inhibiting the retrograde transport of ricin from the endosome to the endoplasmic reticulum; Retro-2 disrupted the functionality of STX5 while exerting no measurable toxicity on mammalian cells. ${ }^{21}$ To initiate our studies with Retro-2, the effect of this molecule on several cell parameters was assessed. Its effects on the distribution of STX5, the Golgi marker GM130, and the plasma membrane markers $\mathrm{CR} 3$ and FCR were evaluated in the presence of increasing concentrations of Retro-2. Retro-2 had no effect on the surface expression of CR3 (Figure 1A). However, with increasing concentrations of Retro-2, FcR was apparently retained in a cellular compartment, although there was no appreciable difference in the surface expression of this molecule (Figure 1B). The effect of Retro-2 on the localization of STX5, compared with GM130, was shown in Figure 1C. All concentrations of Retro-2 had no appreciable effect on GM130 localization; however, increasing concentrations of Retro-2 resulted in the specific dispersal of STX5 from a site that had some overlap with GM130 to a diffuse cellular distribution. Together, these observations suggested that Retro-2 had limited, if any, effect on the trafficking of cell surface molecules and the organization of Golgi. The specific effect of Retro-2 on STX5 distribution was consistent with that of previous studies. ${ }^{21}$

The effect of Retro-2 on activated secretion was also determined by assessing the secretion of IL- 6 from macrophages activated with LPS/interferon- $\gamma$ (Figure 2). There was no significant difference in the secretion of IL-6 with increasing concentrations of Retro-2. These obser- vations suggested that the secretion of molecules, which requires adequate functioning of the secretory pathway, was not affected by Retro-2. Once again, they complemented the observations of Stechmann et al, ${ }^{21}$ who assessed the effect of Retro-2 on several other cellular processes and concluded that the effects of Retro- 2 were restricted to its effect on STX5 function.

\section{Retro-2 Blocked LPV Distention}

In light of previous observations that showed that knocking down STX5 blocked LPV distention and resulted in limited parasite replication, experiments were designed to determine whether Retro-2 would have a disruptive effect on LPV development. These experiments were performed initially on RAW264.7 macrophages. These studies compared the effect of Retro-2 with that of two other molecules from the Chembridge library, which were synthesized using the

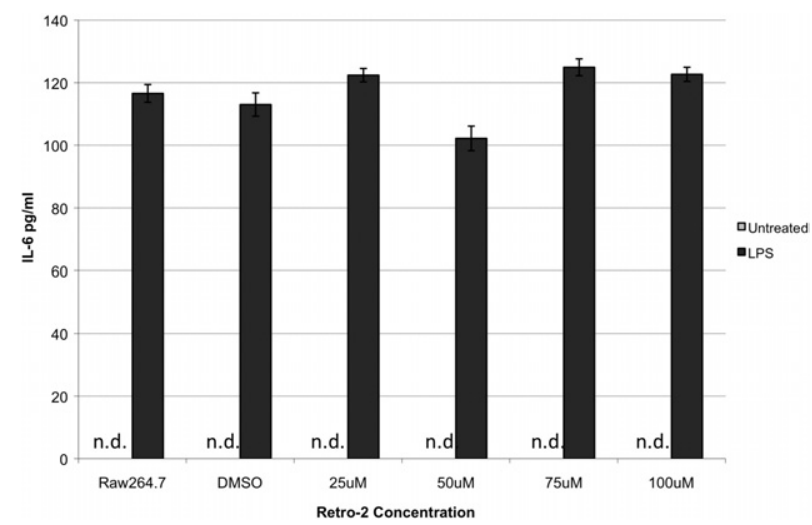

Figure 2. Activated secretion of IL- 6 is not affected by Retro- 2 treatment RAW264.7 cells were treated with Retro- 2 for 2 hours and then interferon- $\gamma$ $(100 \mathrm{U} / \mathrm{mL})$ and LPS $(10 \mathrm{mg} / \mathrm{mL})$ were added to cultures and left for 24 hours. The supernatant fluid was collected, and secreted IL-6 was measured. IL-6 was not detected in 2 hour old infections (n.d.). Data are compiled from three experiments. 

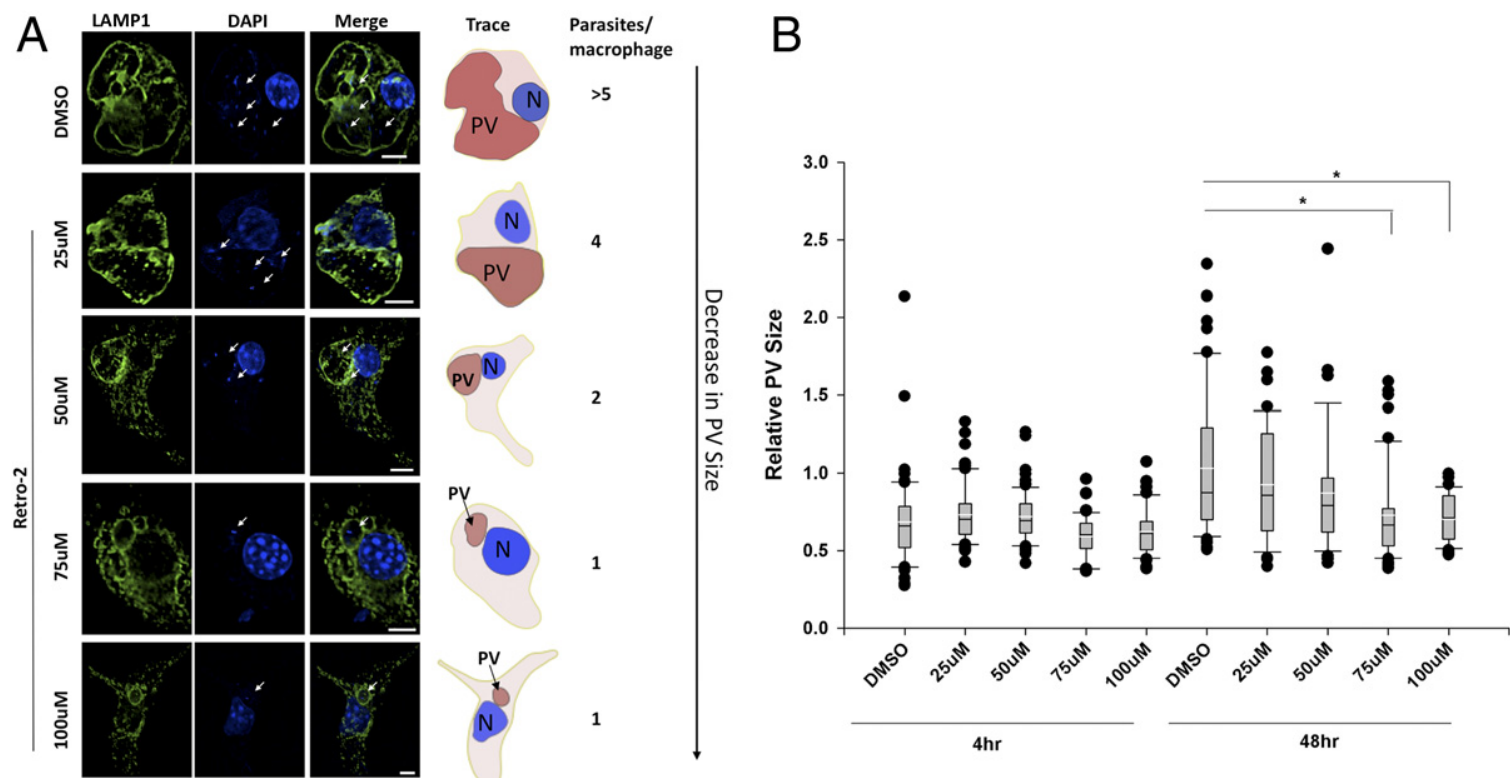

Figure 3. Retro-2 treatment blocks LPV development in primary macrophages. PECs were infected at a parasite/macrophage ratio of 10:1 for 2 hours, and then increasing concentrations of Retro- 2 was added to cultures. Infections were stopped at indicated times, and cells were labeled for visualization of LAMP-1. Representative images of 48-hour infected cells after treatment with the indicated concentration of Retro-2 are shown (A). A trace of labeled cells shows contours of LPVs and host cell nuclei. Arrows, parasites. LPV sizes were obtained and plotted relative to the size of nuclei (B). White lines indicate the mean of the ratios; black lines indicate median of the ratios. Progression of the infection was determined by counting parasites per infected cell in at least 50 infected cells per coverslip for each condition at the indicated times. The data presented were compiled from at least three experiments. The significance of differences was assessed using the $t$-test. ${ }^{*} P \leq 0.05$.

same pharmacophore query approach. The results showed reduced LPV sizes in RAW264.7 cells in the presence of increasing concentrations of Retro-2, compared with LPVs in infected cells incubated with vehicle alone (see Supplemental Figure S1A at http://ajp.amjpathol.org). Moreover, there was no evidence of parasite replication in infected cells in the presence of Retro-2 (see Supplemental Figure S1B at http://ajp.amjpathol.org). Unfortunately, 5322770 and 5322213 were either poorly soluble or toxic to mammalian cells at modest concentrations. We then elected to test the effect of Retro-2 on primary macrophages obtained from PECs. In PECs, LPVs that harbored $L$. amazonensis parasites distended even more extensively to sizes greater than the size of the host cell nucleus; furthermore, by 72 hours after infection (PI), LPVs contained an average of five to six parasites. Retro-2 was added to PECs 2 hours after infections were initiated. The experiments were designed so that the effect of Retro-2 on the infected cell could be evaluated after parasite internalization. The infections were then stopped after an additional 2 hours (4 hours PI) and at 24, 48, and 72 hours PI. Representative examples of 48-hour infected PECs labeled with anti-LAMP-1 are shown in Figure 3A. LPVs were traced to highlight their contours. Retro- 2 caused an apparent reduction in the size of LPVs with increasing concentrations. The sizes of LPVs were measured at the times previously indicated and presented as a ratio of LPV size/size of the infected cell nucleus (Figure 3B). With increasing concentrations of Retro-2, there was a small, but significant, reduction in the size of LPVs after as early as 2 hours after the addition of Retro-2. The effect of Retro-2 on LPV sizes at 48 hours PI was much more impressive. Compared with LPVs in cells treated with vehicle alone, in which LPVs were, on average, 1.1 times the size of the host cell nucleus, treatment with 75 or $100 \mu \mathrm{mol} / \mathrm{L}$ of Retro-2 resulted in LPVs that were approximately half the size of the host cell nucleus. The distribution of LAMP-1 in the infected cells was not affected by Retro-2 treatment; although LPVs were smaller, they were still delineated by a membrane that is LAMP-1 positive.

\section{Retro-2 Inhibited Leishmania Replication in Infected Cells}

Next, the survival and replication of parasites in infected cells were determined. In control cells, there was a steady increase in the number of parasites per macrophage up to an average of 5.5 parasites per macrophage at 72 hours after infection (Figure 4). However, in the presence of 75 or $100 \mu \mathrm{mol} / \mathrm{L}$ Retro-2, there was no increase in the number of parasites per macrophage during that time course. Treatment with Retro-2 blocked the replication of Leishmania parasites within PECs and resulted in a decrease in the number of parasites per macrophage, compared with the number of parasites per macrophage at 4 hours PI.

\section{STX5 Is Not Recruited to LPVs in Retro-2-Treated Cells}

Retro-2 caused a mislocalization or redistribution of STX5 (Figure 1); this might result in limited association of STX5 with its cognate partners, such as sec22b in cells. To determine whether limited association of sec22b and STX5 at the LPV membrane might be part of the mecha- 


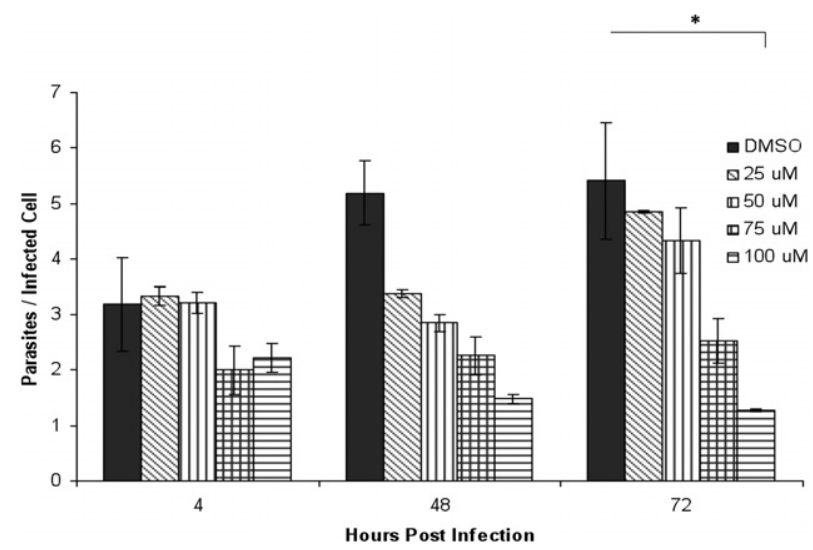

Figure 4. Retro-2 treatment inhibits Leishmania replication in primary macrophages. PECs were infected for 2 hours before increasing concentrations of Retro-2 was added to cultures. Infections were stopped at indicated times, and the number of parasites per infected cell was determined. At least 50 infected cells were scored for each condition at the indicated times. Each data point is compiled from at least three experiments. ${ }^{*} P \leq 0.05$.

nism by which Retro-2 affected LPV development, co-IP experiments were performed. Figure 5A shows that when sec22b is immunoprecipitated from whole lysates of infected cells incubated with vehicle (DMSO) or with Retro-2, comparable amounts of STX5 are co-IP. Other SNARE molecules, specifically D12 and STX18, that associated with sec22b in the ER were also decreased at comparable levels. However, there were differences observed when lysates were obtained from LPV-enriched fractions. After infection for 8 hours in the presence or absence of Retro-2, infected cells were broken mechanically and then the LPVs were enriched by sucrose density fractionation, as previously described. ${ }^{23}$ Infected cells were obtained after 8 hours of infection because recovery of intact LPVs was adversely affected by the continuous distention of LPVs. The LPV-enriched fraction was lysed and processed by the co-IP protocol previously described. Figure 5B shows that, in enriched LPV lysates from cells incubated with Retro-2, STX5 is barely detectable in the pull down, compared with LPV-enriched lysates from cells incubated with vehicle alone. The figure also shows that the association of STX18 with sec22b on the LPV is not affected by Retro-2 treatment. A densitometric scan of these precipitation results is shown in Figure 5C. These observations implied that, although STX5 was redistributed in the Retro-2-treated cells, Retro-2 did not have an inhibitory effect on the interactions of STX5 and sec22b in all cellular compartments.

\section{Retro-2 Treatment Modulated Experimental L. amazonensis Infections}

In light of the observations that Retro-2 blocked LPV development, resulting in limited parasite replication in infected cells, we determined the effect of Retro-2 on the course of an experimental Leishmania infection. These experiments were informed by the observation that administration of Retro- 2 protected mice from up to 150 $\mathrm{mg} / \mathrm{kg}$ of ricin, which was otherwise fatal. ${ }^{21}$ They showed that mice can tolerate up to $400 \mathrm{mg} / \mathrm{kg}$ of Retro-2 without any apparent adverse symptoms, which would be suggestive of toxicity. We evaluated the effect of a single dose of 20 or $100 \mathrm{mg} / \mathrm{kg}$ of Retro-2 administered 1 day after the initiation of infection with stationary phase promastigotes or 3 weeks after initiation of the infection. The later portion of the protocol was to determine whether Retro-2 would have an effect on infections that were already established. As Figure 6A shows, a $20 \mathrm{mg} / \mathrm{kg}$ dose of Retro-2 had no effect on the course of an $L$. amazonensis infection. In contrast, a $100 \mathrm{mg} / \mathrm{kg}$ dose administered either 1 day or 3 weeks after infection significantly altered the course of the Leishmania infection. After 9 weeks of infection, the feet of the infected mice were recovered and the parasites therein were enumerated in limiting dilution assays. As Figure 6B shows, a single treatment of mice with Retro-2 resulted in approximately a log less parasites compared with infected mice that received vehicle alone.

\section{Retro-2 Treatment Has a Direct Effect on Leishmania Replication in Axenic Culture}

To address the likelihood that Retro-2 exerted a direct effect on parasite viability, the growth of Leishmania par-
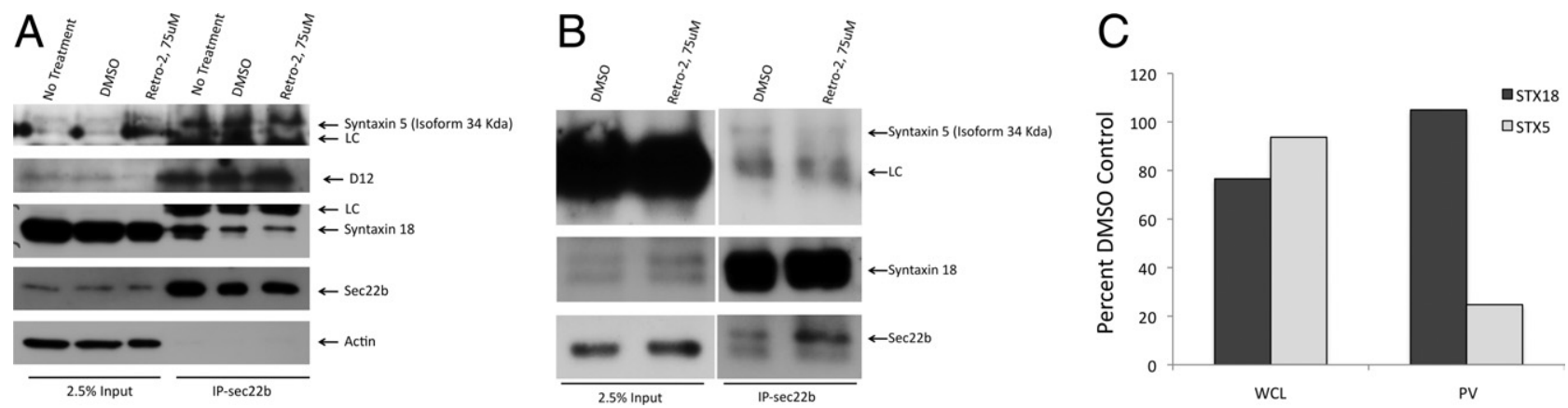

Figure 5. Retro- 2 treatment blocks formation of $S T X 5 / \mathrm{sec} 22 \mathrm{~b}$ complexes at LPV in RAW264.7 cells. RAW264.7 cells were infected for 2 hours before Retro-2 ( 75 $\mu \mathrm{mol} / \mathrm{L})$ was added to the culture. Cells were left for an additional 6 hours before incubating with N-ethylmaleimide at 1 mmol/L in serum-free media for 15 minutes. Cells were lysed, and a co-IP using sec $22 \mathrm{~b}$ antibody was performed on whole cell lysate (A) or the LPV fraction (B). The co-IP was run on SDS-PAGE and transferred to a polyvinylidene difluoride membrane. The membrane was probed initially for STX5; it was then stripped and reprobed for D12, STX18, sec22b, and actin, with stripping after each antibody. C: Densitometric scans of STX5 and STX18 in PVs and whole cell lysate (WCL) from Retro-2-treated cells are presented relative to DMSO control. Densitometry is shown for the image presented. Co-IP/Western blot analysis data are representative of three experiments. 

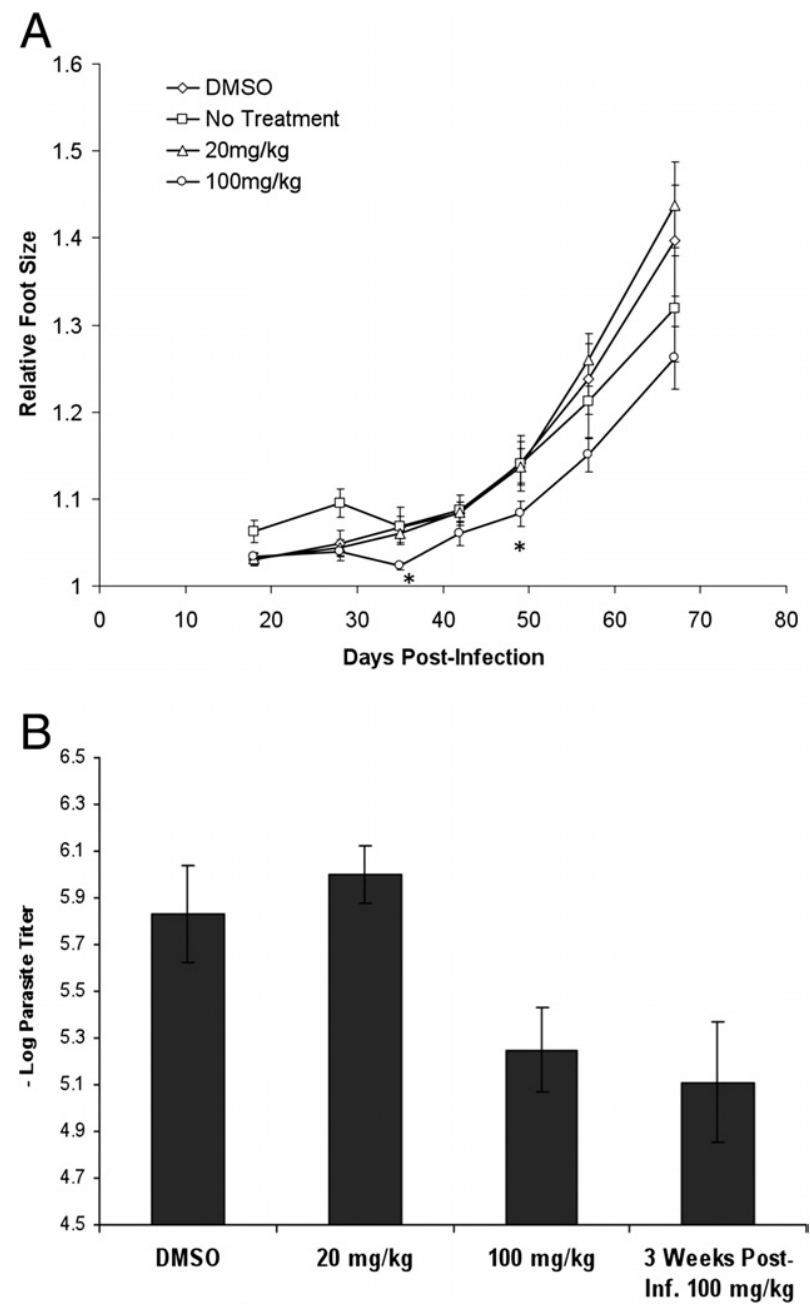

Figure 6. Retro-2 can control the course of an experimental infection. BALB/c mice were administered either 20 or $100 \mathrm{mg} / \mathrm{kg}$ Retro-2 i.p., 24 hours after infection with $2 \times 10^{6}$ stationary stage promastigotes. A separate group of mice was administered the $100 \mathrm{mg} / \mathrm{kg}$ dose 3 weeks after the infection was initiated. Control mice were injected with an equivalent amount of DMSO. Foot sizes of treated mice were obtained at the indicated days after infection. The mean foot sizes are plotted (A). After a 9-week infection course, the infected feet from all of the mice were recovered and the parasite density per foot was determined by limiting dilution. The mean titer of parasites from each group is plotted (B). Each group consisted of 8 to 12 mice. Asterisk, significant difference compared with mice that were treated with vehicle alone.

asites in axenic cultures was determined over a 9-day growth period. As Figure 7 shows, at 50, 75, and 100 $\mu \mathrm{mol} / \mathrm{L}$ concentrations, Retro-2 inhibited the growth of parasites. Parasites remained viable during the early days in the presence of Retro-2; however, they were unable to replicate. Unlike mammalian cells that were able to replicate in the presence of Retro-2, Leishmania parasites were apparently disproportionately more sensitive to this small molecule that targets STX5 function.

\section{Discussion}

In mammalian cells, Leishmania parasites reside in LPVs that are connected with the endomembrane system of the host cell that connects vesicles and organelles. The stud- ies herein show that Retro-2, a small molecule that targets a component of the machinery that mediates fusion of LPVs with a subset of early secretory vesicles, can limit the development of LPVs. Furthermore, the presence of Retro-2 results in the control of Leishmania infections in both in vitro and in vivo settings. Significantly, although Retro-2 caused mislocalization or redistribution of STX5, it had limited adverse effects on the physiological characteristics of host macrophages and the intact experimental host. An unexpected, but welcome, observation was that Leishmania parasites are susceptible to Retro-2 treatment. This later observation implies that the control of Leishmania infections by Retro-2 was achieved by both direct and indirect effects on the parasite.

Retro-2 was identified by Stechmann et $\mathrm{al}^{21}$ in studies that aimed at the identification of molecules that can mitigate the toxicity of ricin. The molecules that emerged from that screen, Retro-1 and Retro-2, disrupted the intracellular trafficking of ricin and consequently blocked ricin toxicity. Our previous studies had shown that, in infected cells exposed to ricin, ricin trafficked into LPVs via a brefeldin A-sensitive pathway. ${ }^{6}$ Moreover, disruption of STX5 function in knockdown experiments resulted in inhibition of LPV development. ${ }^{7}$ In light of those observations, we hypothesized that Retro- 2 should have an effect on the development of LPVs. The observations above demonstrated that the redistribution of STX5 by Retro-2 treatment resulted in limited availability of STX5 for the development of LPVs, even though the availability of STX5 for pairing with sec22b was not globally affected. The co-IP experiments explained, in part, why there was minimal toxicity associated with the treatment of cells with Retro-2. Our observations on the absence of Retro-2 toxicity were consistent with the conclusion of Stechmann et $\mathrm{al}^{21}$ who also assessed the effects of Retro- 2 on a range of other cellular processes.

A single administration of Retro-2 resulted in a significant reduction of both early and ongoing infections. We elected to evaluate Retro-2 after a single treatment in large part because this was not a drug study that would

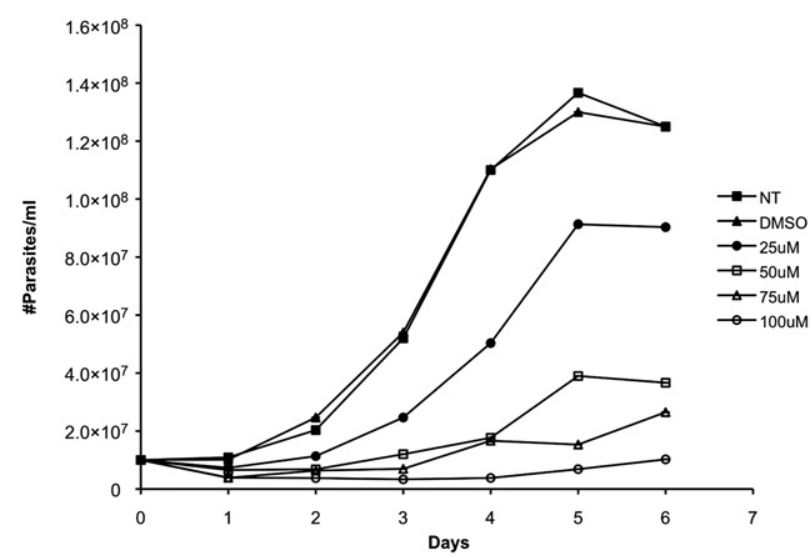

Figure 7. Retro- 2 inhibits parasite replication in axenic cultures. The effect of Retro-2 on L. amazonensis parasites grown in Schneider's culture medium is shown. Parasites were cultured in the presence of the indicated amounts of Retro-2, the vehicle DMSO or left untreated (NT); small aliquots were sampled at the indicated times, and the parasite count was obtained. Cultures are grown in triplicate, and the experiment is performed twice. 
be intended to determine the dosage at which Retro-2 would be effective; instead, the single treatment allowed us to more straightforwardly assess the effect of Retro-2. The studies with Retro-2 clearly demonstrate that small molecules can be derived with specificity to an individual SNARE that can control a pathogen infection. The greater challenge will be to derive the appropriate assay in which to evaluate molecules that can target SNAREs that mediate the development of this and other pathogen-containing compartments.

An unexpected, but welcome, surprise with Retro-2 was the fact that Leishmania parasites were sensitive to the molecule. A report on the analysis of SNAREs in the Leishmania genome had described the presence of an STX5 homologue. ${ }^{24}$ Although a functional analysis of Leishmania SNAREs was not performed, the authors of that article stated that some classes of SNAREs appeared to be missing from the Leishmania genome; they reasoned that, in the absence of alternative SNAREs, Leishmania might be less able to overcome perturbations in SNARE function. Similar observations on the limited repertoire or diversity of SNAREs in other lower eukaryotes, compared with that observed in mammalian cells, have been made. ${ }^{25}$ In light of the fact that Retro-2 was not toxic to their mammalian host cells, the direct effect of Retro-2 on parasite viability increased the likelihood that targeting parasite SNAREs could become a viable strategy for controlling Leishmania infections.

\section{References}

1. Scianimanico S, Desrosiers M, Dermine JF, Meresse S, Descoteaux A, Desjardins M: Impaired recruitment of the small GTPase rab7 correlates with the inhibition of phagosome maturation by Leishmania donovani promastigotes. Cell Microbiol 1999, 1:19-32

2. Duclos S, Diez R, Garin J, Papadopoulou B, Descoteaux A, Stenmark $H$, Desjardins M: Rab5 regulates the kiss and run fusion between phagosomes and endosomes and the acquisition of phagosome leishmanicidal properties in RAW 264.7 macrophages. J Cell Sci 2000, 113:3531-3541

3. Lang T, Hellio R, Kaye PM, Antoine J-C: Leishmania donovani-infected macrophages: characterization of the parasitophorous vacuole and potential role of this organelle in antigen presentation. J Cell Sci 1994, 107:2137-2150

4. Russell DG, Xu SM, Chakraborty P: Intracellular trafficking and the parasitophorous vacuole of Leishmania Mexicana infected macrophages. J Cell Sci 1992, 103:1193-1210

5. Gagnon E, Duclos S, Rondeau C, Chevet E, Cameron PH, SteeleMortimer O, Paiement J, Bergeron JJ, Desjardins M: Endoplasmic reticulum-mediated phagocytosis is a mechanism of entry into macrophages. Cell 2002, 110:119-131

6. Ndjamen B, Kang BH, Hatsuzawa K, Kima PE: Leishmania parasitophorous vacuoles interact continuously with the host cell's endoplas- mic reticulum: parasitophorous vacuoles are hybrid compartments. Cell Microbiol 2010, 12:1480-1494

7. Canton J, Ndjamen B, Hatsuzawa K, Kima PE: Disruption of the fusion of Leishmania parasitophorous vacuoles with ER vesicles results in the control of the infection. Cell Microbiol 2012, 14:937-948

8. Cai H, Reinisch K, Ferro-Novick S: Coats, tethers, Rabs, and SNAREs work together to mediate the intracellular destination of a transport vesicle. Dev Cell 2007, 12:671-682

9. Jahn R, Scheller RH: SNAREs: engines for membrane fusion. Nat Rev Mol Cell Biol 2006, 7:631-643

10. Fasshauer D, Sutton RB, Brunger AT, Jahn R: Conserved structural features of the synaptic fusion complex: SNARE proteins reclassified as Q- and R-SNAREs. Proc Natl Acad Sci U S A 1998, 9:15781-15786

11. Atlashkin V, Kreykenbohm V, Eskelinen EL, Wenzel D, Fayyazi A, Fischer von Mollard G: Deletion of the SNARE vti1b in mice results in the loss of a single SNARE partner, syntaxin 8. Mol Cell Biol 2003, 23:5198-5207

12. Gordon DE, Bond LM, Sahlender DA, Peden AA: A targeted siRNA screen to identify SNAREs required for constitutive secretion in mammalian cells. Traffic 2010, 11:1191-1204

13. Kunwar AJ, Rickmann M, Backofen B, Browski SM, Rosenbusch J, Schöning S, Fleischmann T, Krieglstein K, Fischer von Mollard G: Lack of the endosomal SNAREs vti1a and vti1b led to significant impairments in neuronal development. Proc Natl Acad Sci U S A 2011, 108:2575-2580

14. Becker T, Volchuk A, Rothman JE: Differential use of endoplasmic reticulum membrane for phagocytosis in $\mathrm{J} 774$ macrophages. Proc Natl Acad Sci U S A 2005, 102:4022-4026

15. Hatsuzawa K, Tamura T, Hashimoto H, Hashimoto H, Yokoya S, Miura M, Nagaya $H$, Wada I: Involvement of syntaxin 18, an endoplasmic reticulum (ER)-localized SNARE protein, in ER-mediated phagocytosis. Mol Biol Cell 2006, 17:3964-3977

16. Hatsuzawa K, Hashimoto H, Hashimoto H, Arai S, Tamura T, HigaNishiyama A, Wada I: Sec22b is a negative regulator of phagocytosis in macrophages. Mol Biol Cell 2009, 20:4435-4443

17. Arasaki K, Roy CR: Legionella pneumophila promotes functional interactions between plasma membrane syntaxins and Sec22b. Traffic 2010, 11:587-600

18. Cebrian I, Visentin G, Blanchard N, Jouve M, Bobard A, Moita C, Enninga J, Moita LF, Amigorena S, Savina A: Sec22b regulates phagosomal maturation and antigen crosspresentation by dendritic cells. Cell 2011, 147:1355-1368

19. Barbier J, Bouclier C, Johannes L, Gillet D: Inhibitors of the cellular trafficking of ricin. Toxins (Basel) 2012, 4:15-27

20. Wahome PG, Bai Y, Neal LM, Robertus JD, Mantis NJ: Identification of small-molecule inhibitors of ricin and shiga toxin using a cell-based high-throughput screen. Toxicon 2010, 56:313-323

21. Stechmann B, Bai SK, Gobbo E, Lopez R, Merer G, Pinchard S, Panigai L, Tenza D, Raposo G, Beaumelle B, Sauvaire D, Gillet D, Johannes L, Barbier J: Inhibition of retrograde transport protects mice from lethal ricin challenge. Cell 2010, 141:231-242

22. Soong L, Duboise SM, Kima P, McMahon-Pratt D: Leishmania pifano amastigote antigens protect mice against cutaneous leishmaniasis. Infect Immun 1995, 63:3559-3566

23. Kima PE, Dunn W: Exploiting calnexin expression on phagosomes to isolate Leishmania parasitophorous vacuoles. Microb Pathog 2005 , 38:139-145

24. Besteiro S, Coombs GH, Mottram JC: The SNARE protein family of Leishmania major. BMC Genomics 2006, 7:250

25. Kienle N, Kloepper TH, Fasshauer D: Differences in the SNARE evolution of fungi and metazoa. Biochem Soc Trans 2009, 37:787791 